\section{Training interventions on Helping Babies Breathe among health workers in tertiary hospital of the Republic of South Sudan: A non-randomized quasi-experimental study}

\author{
Christopher Vunni Draiko, ${ }^{1}$ \\ Khemika Yamarat, ${ }^{1}$ Alessio Panza, ${ }^{1}$ \\ Judith Draleru, ${ }^{2}$ Martin Taban, ${ }^{3}$ \\ Joseph Onyango, ${ }^{3}$ Regina Akur, ${ }^{2}$ \\ Rose Aliru Omega ${ }^{4}$
}

${ }^{1}$ College of Public Health Sciences, Chulalongkorn University, Bangkok, Thailand; ${ }^{2}$ Juba College of Nursing and Midwifery, Juba Teaching Hospital, Juba, South Sudan; ${ }^{3}$ Real Medicine Foundation, Kampala, Uganda; ${ }^{4}$ United Nation Population Fund, Juba, South Sudan

\begin{abstract}
This study aimed to examine the effects of the Helping Babies Breathe (HBB) training interventions program on the knowledge, psychomotor skills, and competency of health workers in managing birth asphyxia and reducing mortality of newborns experiencing asphyxia within 24 hours. This study used pre- and post-test design (quasi experimental study). Purposive sampling was employed, and a computer-generated number was used to select the participants. Health workers from Juba Teaching Hospital comprised the intervention group. They were evaluated before and after the training from February to June 2017. A post training skill and competency evaluation was performed using a NeoNatalie newborn simulator and was repeated after three months of implementation for intervention and control group. Seventy health workers were enrolled; 40 were in the intervention group and 30 in the control group. Early newborn mortality due to asphyxia within 24 hours in intervention and control measure at pre and post implementation showed a significant reduction within the intervention than the control. Knowledge, psychomotor and competency of health care workers improved immediately after training and early newborn mortality reduced by half at the end of three months. It is recommended that training of health workers on HBB should be scaled up in most of the health facilities in South Sudan.
\end{abstract}

\section{Introduction}

South Sudan has a high number newborn mortality. The neonatal mortality was estimated at 35 per 1,000 live births with infant mortality of 67 per 1,000 live births. ${ }^{1}$

In $2015,4.5$ million (75\%) of all underfive deaths occurred within the first year of life. The risk of a child dying before completing the first year of age was highest in the African region (55 per 1000 live births) and over five times higher than that in the developed region (10 per 1000 live births). Although, there is reported decreased in the number of infant mortality birth worldwide, more newborn deaths are reported in low and poor developing countries where access to health care for the vulnerable (women and newborn) was difficult and sometimes nonexistent. $^{2}$

According to the World Health Organization $^{3}$ an estimated 136 million infants are born each year, and this figure is expected to rise globally to nearly $137 \mathrm{mil}-$ lion births yearly by 2016. In the entire human life cycle, the riskiest period is the day of birth ${ }^{4}$ previous studies reported that 136 of the one million of newborn births each year will not survive their first day of life. ${ }^{5}$ Based on the global total of one million deaths each year, efforts must be aimed at reducing the high number of newborn deaths.

The main causes of newborn deaths have remained unchanged for the last decade and are usually infection-related complications (26\%), intrapartum complications (24\%) including birth asphyxia, preterm delivery (34\%) with breathing problems contributing to mortality and morbidity, and congenital abnormalities (9\%). ${ }^{6}$ The real cause of newborn deaths is difficult and most challenging to determine for the health professionals. Nurses, midwives, clinical officers, medical officers, maternal health workers, and community health workers attending births must have the essential knowledge and skills to assess the neonates' breathing status and effectively respond as needed. Majority of the health workers/staff often failed to recognize breathing difficulties early and, most of the times, could not assess and take immediate action to resuscitate non-breathing babies at the time of delivery. Priority has often been given to the mother's needs. The neonates often remained unattended for several minutes with little attention. This problem could be partly attributed to not having a staff member present dedicated to the newborns. Despite the burden of newborn death being high in the low and poor countries, the coverage of skilled birth attendance is very scarce in these countries. ${ }^{7}$ Similarly,
Correspondence: Christopher Vunni Draiko, College of Public Health Sciences, Chulalongkorn University, Bangkok, Thailand.

E-mail: chrissvunni@gmail.com

Key words: Asphyxia, Babies breath; Resuscitation; Neonatal mortality; Psychomotor skills.

Acknowledgements: the authors want to thank all the health workers who diligently participated in the study, Ministry of Health and the staff of Juba and Wau teaching Hospital. Their sincere thank goes to the staff of Juba College of Nursing and Midwifery providing room for the training and the United Nations Midwives who supported the training.

Contributions: CVD designed the study, interpreted data and wrote the report. KY, AP, JD, MT, JO, RA, RAO supported the study, reviewed the manuscript and proofread the article before submission.

Conflict of interest: the authors declare no potential conflict of interest.

Funding: none.

Received for publication: 13 January 2018. Accepted for publication: 25 June 2018.

This work is licensed under a Creative Commons Attribution 4.0 License (by-nc 4.0).

(C) Copyright C.V. Draiko et al., 2018

Licensee PAGEPress, Italy

Healthcare in Low-resource Settings 2018; 6:7276 doi:10.4081/hls.2018.7276

more than one million preterm neonates die from complications of preterm delivery, including respiratory distress syndrome, as majority of the preterm newborns require assistance to initiate breathing at birth. ${ }^{4}$

Having known that prematurity and intrapartum hypoxic are the main causes of early neonate mortality in majority of the neonates, global, regional, and local training of health workers in basic neonatal resuscitation will help improve the newborn survival and will save hundreds of thousands of newborn infants yearly. ${ }^{4}$

Despite the benefits of the health workers' training on neonatal resuscitation in averting newborn deaths, the coverage of neonatal resuscitation remains very low in settings with a high burden of neonatal deaths. Currently, various neonatal resuscitation training courses are being promoted and implemented in many countries in order to build the capacity and competence of the health workers to become better qualified in managing sick newborns or children in the emergency setting. 
A number of guidelines and algorithms exist, and most of these can be found online. However, most of the guidelines and algorithms are reported to have been based on the consensus of the pediatricians' expertise rather than the evidence-based training module. ${ }^{8}$

Newborn life support, neonatal resuscitation program, the WHO's essential newborn care, and pediatric life support are the courses used in teaching neonatal resuscitation. A review conducted by Opiyo and English $^{9}$ found inadequate evidence to prove that the in-service training in neonatal resuscitation had improved the skill and performance of the health workers in caring for critically ill newborn baby. However, there is still some evidence to show the benefits of training of health workers in newborn resuscitation.

Among the most effective strategies available for low-resource setting is the Helping Babies Breathe (HBB) program, a neonatal resuscitation training program aimed to increase the knowledge and skills of skilled birth attendants (SBA) ${ }^{10}$ The HBB is a life support program developed by the American Academy of Pediatrics in collaboration with other organizations. ${ }^{11}$ With the objective of achieving a sustainable development goal of ending preventable neonatal and under-five child deaths through increasing the coverage of SBA and improving the quality of maternal and newborn care, the training intervention on Helping Babies Breath in hospitals and health centers is essential. The "HBB-plus" training intervention is believed to increase the knowledge, skill, and competency of the health workers on newborn resuscitation that will contribute to reducing asphyxia-related newborn mortalities in South Sudan.

Neonatal resuscitation is not new in the South; however, the simplified version of resuscitation packaged as Helping Babies Breathe is a new idea that has not been explored and implemented in the public health facilities in South Sudan on large scale. The training was adapted from the Helping Babies Breathe program developed by the American Academy of Pediatricians for low-resourced countries. This study aims to evaluate the immediate and longterm effects of the HBB training on the knowledge and skills of health professionals about neonatal resuscitation and the reduction of neonatal mortality due to asphyxia.

\section{Materials and Methods}

The protocol for this non-randomized clinical trial and supporting Trend statement checklist are available as supporting information respectively and the registration was registered retrospectively. Reason for late registration: the study was initially measuring the increase in the knowledge, skill, and competency, and the distal portion of the impact on newborn resuscitation and survival was added later.

\section{The study population and design}

The pre and post study was conducted two tertiary hospital of Juba and Wau in South Sudan. After obtaining ethical clearance from the South Sudan Ethical review board, Ministry of Health, and Administrator of both Juba and Wau Teaching Hospital, a total of 70 health workers (40 from the intervention group and 30 from the control group) comprising midwives, clinical officers, nurses, community maternal health workers, and intern doctors were selected. The 40 health workers from Juba Teaching Hospital were trained on simplified Helping Babies Breathe for two days. Purposive sampling was used in selecting the participants of the study. The implementation period lasted for three months from March to June 2017, and the three months evaluation took place in June. Blinding of the participants was unachievable based on the nature of the intervention. Health workers in the control group never received any HBB training and continued to provide routine care to the newborn, whereas those in the intervention group received two days, 6 hours training based on the HBB protocol.

\section{Participant's recruitment}

Health workers who consented to participate in the study were randomly selected. Forty participants were selected from Juba teaching hospital (Intervention) and thirty from Wau teaching hospital (Control). All health worker selected to participate in the study had no received any training on helping babies breathe protocol for the past one year. Training for the health workers in the intervention lasted for two day six hours each.

\section{Procedures and intervention}

\section{HBB facilitators and participants}

Training of HBB was conducted by experienced facilitator who were trained midwives and received training as research assistants. Training session of the health workers were divided into two phases to allow a ratio of one trainer to 6 participant's trainee and adequate time for supervision. Training covered main area of preparation for birth, newborn routine care, the golden minute and ventilation of the newborn. The participant were introduced to each section of the HBB protocol followed by demonstration and return demonstration by the participants. The trainers and the trainees reviewed the training and practical sessions related to newborn asphyxia, routine care, ventilation. The health workers practiced on NeoNatalie new born simulator. Health workers trained were provided with the basic equipment's for the practice of HBB neonatal resuscitation during and after the training. Participants were asked to evaluate the training session using Likert scale of one to five and majority agreed that the training enhanced their psychomotor skills and competency in newborn resuscitation.

\section{HBB knowledge and psychomotor skills and competency evaluation}

Health workers were tested using multiple choice questionnaire pre and post intervention for both groups lasting for twenty minutes. Psychomotor skill and competency for the intervention and control group were conducted in simulated environment using Bag and Mask checklist for psychomotor skill and OSCEA \&B for competency. Health workers in the intervention group were assessed at pre, post and 3 months period while those in the control group were assessed at posttest and 3 months follow up. Health were scored for each of the steps and action in the checklist. The correctly performed action was awarded a score of one whole incorrect steps or answer was given zero marks. To qualify to have adequate knowledge psychomotor skill and competency for newborn resuscitation, health workers must score $80 \%$ or above and this was also considered successful completion of the course.

\section{Study outcomes}

The primary objective was that $\mathrm{HBB}$ training will improve the knowledge and practical skill of the participants. This was determined tests conducted to evaluate changes before training, post training and 3 months follow up. The secondary was reduction in early neonatal deaths within 24 hours and the observed outcome was tested by Pearson Chi square for newborn deaths due to asphyxia within 24 hours.

\section{Data management and analysis}

Data from the answer sheets and evaluation checklists were entered into SPSS version 20 software, where the results of the pre- and post-test as well as the three-month follow up was analyzed and presented. To get the result desired, all the files containing the different measurements were merged at one point in time before conducting the complete analysis. The mean scores in knowledge, psychomotor skills, and compe- 
tency from the pre- and post-test within the intervention and within control groups was tested using repeated measure ANOVA for within intervention and control group. The unpaired sample t-test was used to test the mean difference in knowledge, psychomotor skills, and competency between the intervention and control group. The result analyzed and obtained was presented and reported as mean of the correctly passing scores + standard deviation. The study was considered significant at $\mathrm{p}$ value $<0.05$.

\section{Ethical Clearance}

The study was approved by Chulalongkorn University College of Public Health, Bangkok, Thailand in December 2016. The ethical clearance for study was approved by the Ethical Review Board, Ministry of Health, and South Sudan in February 2017, and the ethics review committees of Juba and Wau Hospital. Informed consent both written and verbal was obtained from the participants before the training intervention. Verbal approval was sought from the mothers with newborn with asphyxia by the health worker.

\section{Results}

A total of 70 health care workers enrolled in this study of which 40 received simplified HBB training and completed $(100 \%)$ the pre- and post-test course assessment. Approximately 30 participants in the control group took the post-test simultaneously with those of the intervention group. Because the health workers at the control group were unable to receive the training due to insecurity, their pre-training assessment (baseline) for the practical skill was conducted at the time of the post-test administered among the intervention group; it was then considered as the immediate post intervention assessment to evaluate the knowledge, skills, and competency of these health workers. At three months, two of the participants in the intervention group and one from control group were lost to follow up. Therefore, only (67) $96 \%$ of the health workers completed the assessment at threemonth follow up (Figure 1).

\section{Socio demographic and professional characteristics}

Majority of the health workers were aged between 25 years to 35 years. Nurses and midwives were the majority in intervention and control group and were predominantly female $(82.5 \%)$ in intervention and $80 \%$ ) providing newborn care (Table 1). Most of the health workers were working in maternity labor room) 23 (57.5\%) and $19(63.3 \%)$, children ward $16(40 \%)$ and $7(23.3 \%)$ and operating theater $1(2.5 \%)$ and $4(13.3 \%)$ respectively. Majority of the health workers self-reported to be registered nurses and midwives with tertiary and college education $(77.5 \%$ of the intervention versus $73.3 \%$ in control).

The level of income in the middle income bracket of 1001-2,000 SSP varies between intervention and control group with control group slightly receiving higher income compared to intervention. The difference however is not significant.

The duration of practice among the health workers ranged from less than one year to over five years with most having practiced over five years (32.5\%) intervention and versus $(36.7 \%)$ in control group and there was insignificant difference between intervention and control group (Table 1).

\section{Health workers HBB knowledge}

Examining the test mean score of the participants within the intervention and within the control at pretest, posttest intervention and three months of study period tested by repeated measures ANOVA, in intervention, there was significant increase in terms of knowledge between the pretest and immediate post intervention (mean difference increase of $55.2(50.9-59.6) \mathrm{p}<0.05)$ and this decreased slightly between the immediate post intervention and three months follow up with mean difference of 13.3(-17.7-8.87), $\mathrm{p}<0.05)$. This mean decrease between the immediate post intervention and 3 months follow was insignificant.

In the control group, there was slight increase in knowledge between pretest and immediate post (mean difference of 3.1(3.0-9.4) $\mathrm{p}>0.05)$ and this increased further at 3 months follow up (mean difference of $0.3(-0.1-6.0) \mathrm{p}>0.05)$ which was insignificant.

In terms of outcome between the intervention and control group, there was no significant difference in knowledge at baseline $(p>0.05)$. However, this significantly increased in intervention at posttest and 3 months $(\mathrm{p}>0.05)$ (Table 2).

\section{Health workers HBB psychomotor skills}

Repeated measures ANOVA was used to test for the psychomotor skill for the intervention and control group at baseline,

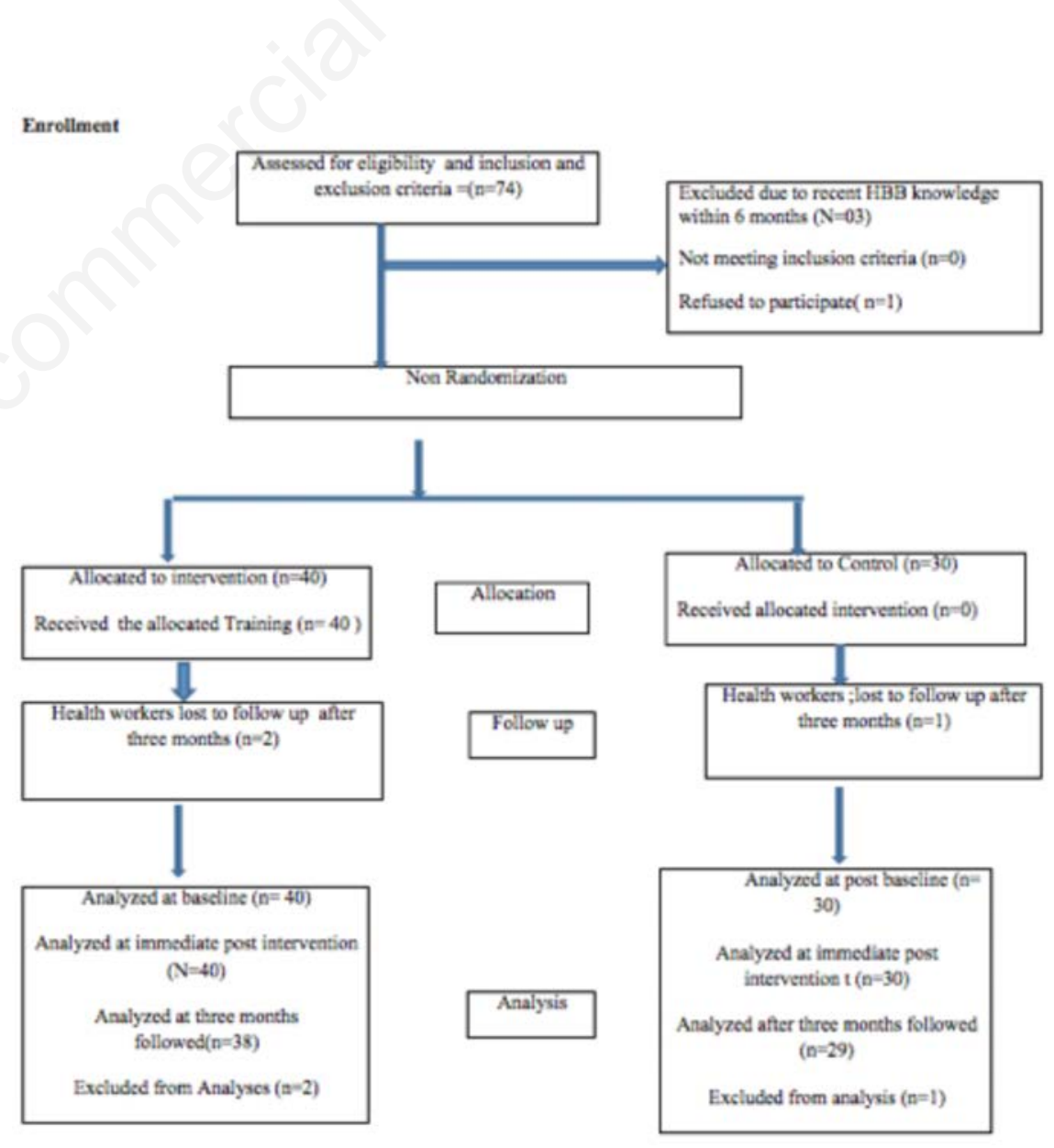

Figure 1. Flow chart for recruitment and allocation health workers. 
immediate post intervention and 3 months follow up (Table 2). When compared from baseline to 3 months follow up, the intervention had significant increase between base line and immediate post intervention with mean difference of $69.2(62.8-75.7)$ $\mathrm{p}<0.05$ and this increased at 3 months follow up (mean difference 0.1(-0.3-0.8), $\mathrm{p}<0.05$ ). In the control group, the baseline was not tested and only the immediate post intervention and 3 months follow-up was tested. From the outcome, there was no significant increase in psychomotor skill in the control (mean difference -3.4 (-11.0-4.10, $\mathrm{p}>0.05$.). Between the intervention and control group at immediate post intervention and 3 months follow up, there was significant increase in psychomotor skill in the intervention compared with the control group $(\mathrm{p}<0.05)$.

\section{Health worker competency for simple resuscitation measured through OSCE A}

In Table 2 below, health workers competency for simple resuscitation in the intervention and control group tested by repeated measures ANOVA at baseline, immediate post intervention and 3 months follow showed that within the intervention group, there was significant increase of competency for simple neonatal resuscitation from baseline and immediate post intervention (mean difference of 61.2(57.0-66.5 $\mathrm{p}<0.05$ ) and deceased slightly at 3 months follow up (mean difference 0.3.1(-4.6-5.32). However, the mean difference between the immediate post intervention and 3 months was not statically significant ( $\mathrm{p}>0.05)$.

Health workers in control group were not tested at baseline for simple resuscita- tion but tested at immediate post intervention and 3 months. Result showed that there was no significant increase in competency of health workers between immediate post intervention and 3 months follow-up $(\mathrm{p}>0.05)$.

\section{Health worker competency complex neonatal resuscitation measured through OSCE B}

When compared from baseline to 3 months of follow up, the intervention group had significant increase between baseline and immediate post intervention $(\mathrm{p}<0.05)$ and this decreased at 3 months follow up (mean difference 2.74(-6.71-1.22) but the changes remained insignificant $(\mathrm{p}>0.05)$

In the control group, the health worker were tested at immediate post intervention and 3 months follow up and there was no sig-

Table 1. Sociodemographic and professional characteristics (age, gender, and educational level).

\begin{tabular}{|c|c|c|c|c|c|c|c|c|}
\hline \multirow{2}{*}{\multicolumn{2}{|c|}{ Demographic Characteristics }} & \multicolumn{2}{|c|}{ Intervention } & \multicolumn{2}{|c|}{ Control } & \multirow{2}{*}{\multicolumn{2}{|c|}{ p- value }} & \multirow[b]{2}{*}{ Statistical test } \\
\hline & & $\begin{array}{l}\text { Freq. } \\
(\mathrm{N}=40)\end{array}$ & $(\%)$ & $\begin{array}{l}\text { Freq. } \\
(\mathrm{N}=30)\end{array}$ & $(\%)$ & & & \\
\hline Age in years & $\begin{array}{l}25-35 \\
36 \text { above }\end{array}$ & $\begin{array}{l}25 \\
15\end{array}$ & $\begin{array}{l}62.5 \\
37.5\end{array}$ & $\begin{array}{l}20 \\
10\end{array}$ & $\begin{array}{l}66.7 \\
33.3\end{array}$ & 0.130 & 0.719 & Chi-square \\
\hline Gender & $\begin{array}{l}\text { Male } \\
\text { Female }\end{array}$ & $\begin{array}{c}7 \\
33\end{array}$ & $\begin{array}{l}17.5 \\
82.5\end{array}$ & $\begin{array}{c}6 \\
24\end{array}$ & $\begin{array}{l}20.0 \\
80.0\end{array}$ & 0.071 & 0.790 & Chi-square \\
\hline Education level & $\begin{array}{l}\text { Primary eight } \\
\text { Secondary } \\
\text { College/ tertiary } \\
\text { Diploma in Midwifery } \\
\text { Community H.W Training }\end{array}$ & $\begin{array}{c}6 \\
3 \\
31 \\
1 \\
1\end{array}$ & $\begin{array}{c}15.0 \\
7.5 \\
77.5 \\
2.5 \\
2.5\end{array}$ & $\begin{array}{c}4 \\
4 \\
22 \\
0 \\
0\end{array}$ & $\begin{array}{c}13.3 \\
13.3 \\
73.3 \\
0 \\
0\end{array}$ & 0.748 & 0.781 & Fisher Exact \\
\hline Professional qualification & $\begin{array}{l}\text { Nurse } \\
\text { Midwives } \\
\text { Maternal Child health officer } \\
\text { Nurse practitioner } \\
\text { Clinical officer } \\
\text { Community Health workers } \\
\text { Skilled birth attendants } \\
\text { Intern Doctor }\end{array}$ & $\begin{array}{c}12 \\
17 \\
3 \\
1 \\
2 \\
4 \\
1\end{array}$ & $\begin{array}{c}30.0 \\
42.5 \\
7.5 \\
2.5 \\
5.0 \\
10.0 \\
2.5\end{array}$ & $\begin{array}{l}10 \\
11 \\
1 \\
0 \\
2 \\
2 \\
1 \\
3\end{array}$ & $\begin{array}{c}33.3 \\
36.7 \\
3.3 \\
0 \\
6.7 \\
6.7 \\
3.3 \\
10.0\end{array}$ & 5.690 & 0.623 & $\begin{array}{l}\text { Fisher Exact } \\
\text { Fisher Exact }\end{array}$ \\
\hline Primary area & $\begin{array}{l}\text { Newborn care } \\
\text { Sick children ward } \\
\text { Maternal and newborn care } \\
\text { Obstetrics/Obstetrician }\end{array}$ & $\begin{array}{c}11 \\
4 \\
25\end{array}$ & $\begin{array}{l}27.5 \\
10.0 \\
62.5\end{array}$ & $\begin{array}{c}8 \\
1 \\
17 \\
4\end{array}$ & $\begin{array}{c}26.7 \\
3.3 \\
56.7 \\
13.3\end{array}$ & 5.987 & 0.097 & \\
\hline Current place of work & $\begin{array}{l}\text { Maternity ward } \\
\text { Children ward } \\
\text { Operating theater (OT) }\end{array}$ & $\begin{array}{c}23 \\
16 \\
1\end{array}$ & $\begin{array}{c}57.5 \\
40.0 \\
2.5\end{array}$ & $\begin{array}{c}19 \\
7 \\
4\end{array}$ & $\begin{array}{l}63.3 \\
23.3 \\
13.3 \\
\end{array}$ & 4.135 & 0.129 & Fisher Exact \\
\hline Monthly income & $\begin{array}{l}300-1000 \text { SSP } \\
1001-2,000 \text { SSP } \\
2,001 \text { SSP and above }\end{array}$ & $\begin{array}{c}18 \\
14 \\
8\end{array}$ & $\begin{array}{l}45.0 \\
35.0 \\
20.0\end{array}$ & $\begin{array}{c}8 \\
16 \\
6\end{array}$ & $\begin{array}{l}26.7 \\
53.3 \\
20.0\end{array}$ & 2.896 & 0.235 & Chi-square \\
\hline $\begin{array}{l}\text { Duration of practice } \\
\text { Two - three years } \\
\text { Four - five years } \\
\text { Over five years }\end{array}$ & $\begin{array}{l}\leq 1 \text { year } \\
10 \\
5 \\
13\end{array}$ & $\begin{array}{c}12 \\
25.0 \\
12.5 \\
32.5\end{array}$ & $\begin{array}{c}30.0 \\
8 \\
5 \\
11\end{array}$ & $\begin{array}{c}6 \\
26.7 \\
16.7 \\
36.7\end{array}$ & 20.0 & 0.980 & 0.806 & \\
\hline Knowledge & Baseline & 17 & 42.5 & & 48 & & 0.6 & Unpaired test \\
\hline Psychomotor skill & Baseline (pretest) & 10 & 26 & $*$ & $*$ & & & \\
\hline Simple Competency & Baseline (pretest) & 10 & 26.9 & $*$ & $*$ & & & \\
\hline Complex competency & Baseline (pretest) & 7 & 17.5 & $*$ & $*$ & & & \\
\hline
\end{tabular}

Significant level at 0.05 . The 25-35 years in case of age is based on the fact that it represents the youthful age group. Fishers Exact test have been used for cell counts less than $5 .{ }^{*}$ psychomotor skill and competency for simple and complex neonatal resuscitation at baseline cannot be compared due to lack of assessment for control at baseline. 
nificant change in the competency for complex neonatal resuscitation $(\mathrm{p}>0.05)$. Between the intervention and control group, health workers in intervention group showed significant increase in competency compared to control group $(\mathrm{p}<0.05)$ (Table 2$)$.

\section{Early neonatal mortality}

A total of 4981 live births were recorded in the hospital registry; 2127 live births registered before implementation from November 2016 to February 2017, and 2062 after implementation from March to June 2017. All births were attended by the health workers in the study during implementation (Figure 2).

\section{Early newborn death within 24 hours}

Table 3 is the composite summary of early newborn mortality due to asphyxia within 24 hours in intervention and control. When compared at pre and post implementation, there was significant reduction in the intervention than the control group in term of newborn mortality within 24 hours. Newborn mortality reduced from $51.9 \%$ pre implementation to $23.5 \%$ post implementation. The percentage decreased in the control group remained insignificant ( $48.1 \%$ to $48.1 \%$ both pre and post implementation).

The reduction and changes within the pre intervention and post implementation

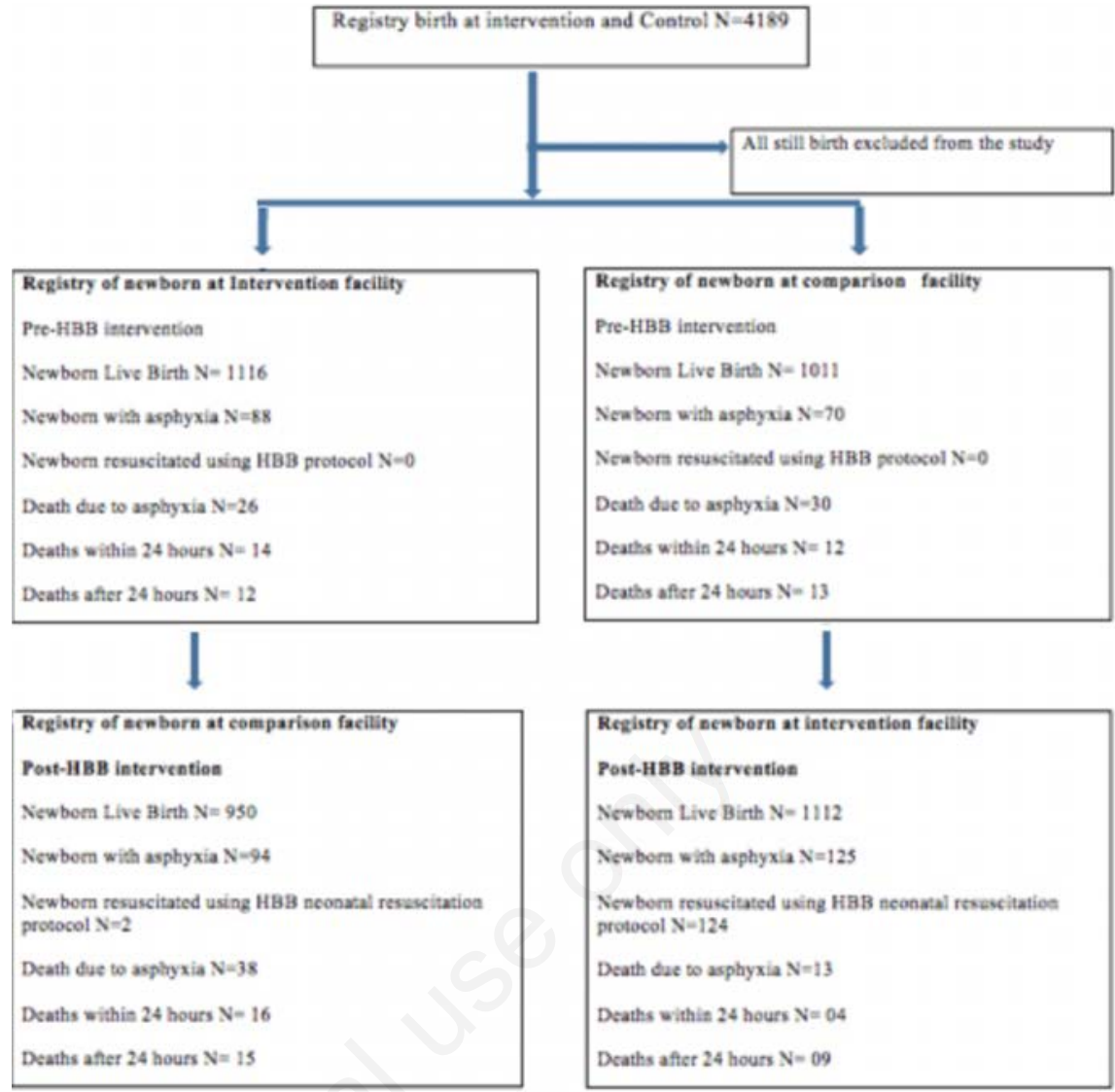

Figure 2. Flow chart for birth registry pre and post implementation.

Table 2. HBB health worker's knowledge, psychomotor skills, competency for simple and complex neonatal resuscitation of the health workers at pretest immediate intervention and 3 moths follow up.

\begin{tabular}{|c|c|c|c|c|c|c|}
\hline & \multicolumn{2}{|c|}{ Intervention } & \multirow[t]{2}{*}{ P-Value $^{a}$} & \multicolumn{2}{|c|}{ Control } & \multirow[t]{2}{*}{ P-Value } \\
\hline & Mean & Mean df. (CI) & & Mean & Mean df. (CI) & \\
\hline \multicolumn{7}{|c|}{ Knowledge } \\
\hline Pre- test & $42.5 \pm 17.3$ & & & $48.0 \pm 13.9$ & & \\
\hline Immediate post intervention & $97.8 \pm 3.4$ & $55.2(50.9-59.6)$ & $<0.001$ & $51.2 \pm 11.2$ & $3.1(-3.0-9.4)$ & 0.9 \\
\hline 3 months follow-up & $84.7 \pm 7.7$ & $-13.3(-17.7-8.87)$ & $<0.001$ & $50.9 \pm 15.7$ & $-0.3(-0.1-6.0$ & 0.9 \\
\hline \multicolumn{7}{|c|}{ Psychomotor skills } \\
\hline Pre-test & $26.1 \pm 19.9$ & & & $-*$ & - & - \\
\hline Immediate post test & $94.4 \pm 8.5$ & $69.2(62.8-75.7)$ & $<0.001$ & $43.8 \pm 16.7$ & & \\
\hline 3 months follow-up & $95.4 \pm 6.8$ & $0.1(-03-08)$ & $<0.001$ & $40.9 \pm 18.9$ & $3.4(11.0-4.10)$ & 0.37 \\
\hline \multicolumn{7}{|c|}{ Competency for simple neonatal resuscitation } \\
\hline Pre- test & $26.9 \pm 14.6$ & & & $-*$ & - & - \\
\hline Intermediate & $88.8 \pm 8.5$ & $61.2(57.0-66.7)$ & $<0.001$ & $38.9 \pm 8.5$ & - & - \\
\hline \multirow[t]{2}{*}{3 months follow-up } & $89.3 \pm 8.1$ & & & & & \\
\hline & 03.1(-4.6-532) & 0.9 & $41.3 \pm 14.4$ & $2.65(-8.42-3.1)$ & 0.36 & \\
\hline \multicolumn{7}{|c|}{ Competency for complex neonatal resuscitation } \\
\hline Pre test & $17.5 \pm 8.9$ & & & $-* *$ & - & - \\
\hline Intermediate & $90.9 \pm 7.1$ & 73.47(69.5-77.36) & $<0.001$ & $36.5 \pm 13.0$ & & \\
\hline 3 months follow-up & $88.3 \pm 10.8$ & $-274(6.71-1.22$ & 0.17 & $33.1 \pm 8.7$ & $-3.63(-8.2-0.93)$ & 0.12 \\
\hline
\end{tabular}

Data are expressed as Mean difference. *Significant level at 0.005 post intervention and 3 months follow up. Pa-Value within intervention group tested by repeated ANOVA, 0.001 and 0.001 between immediate post intervention and 3 months follow up. ${ }^{* *}$ No baseline conducted for control group for bag and mask, OSCE A and B due to logistical problems, and time. 
period on reduction of among the intervention and control group was tested using person chi squared. Within the intervention group, there was significant change ratio of early newborn mortality compared to the control group within the 24 hours after conducting resuscitation $(\mathrm{p}<0.05)$.

\section{Discussion}

The professional background of the health workers in the intervention group who attended the HBB training in Juba Teaching Hospital had similar characteristics, and the working environment of the control group (Wau Teaching Hospital) was situated 100 kilometers away from the intervention site. Both the intervention and control hospitals are teaching hospital with a similar setup and are supported by the government of South Sudan. This generalized the findings on the evaluation made in other health care settings and public hospitals in the country as well other low-income developing countries with a similar setting.

During our study, we administered the OSCE A and B to the intervention group pre-test, post-test, and at the three-month follow-up, and at post-test and three-month follow-up in the control group because of the insecurity which was not safe for the trainers and the participants to undertake the assessment. Frequently, the OSCE A and $\mathrm{B}$ were considered too difficult to be administered to the participants during the pre-training period. Administering the OSCE A and B pre training helped us estab- lish the health workers' skills and competency pre training and aided the design of the educational instruction approach. Based on the pre-assessment of the practical skill and competency of health workers in the intervention group, we tailored support to each of the participant's ability and understanding of the training, and that facilitated the good result at post training. Our study introduced and implemented a quality improvement cycle as a means of improving the knowledge, skills, and competency of the health workers in the long term.

The HBB training implementation contributed to the improvement of the knowledge of the health workers from intervention Hospital after a two-day training. However, the level of knowledge attained at post-training declined at the end of implementation period. Meanwhile there was no significant increase in knowledge among the health worker in the control site.

The study on HBB training in South Sudan has shown that the HBB knowledge MCQ written scores improved by $55.3 \%$ from $42.5 \%$ at pre-training to $97.8 \%$ immediately after post training (pre-test) (Table 2). This result concurred with a similar study in Kenya which has showed that, passing rate of the knowledge MCQ-based test increased from $75 \%$ to $95 \%$ after a similar training intervention. The use of the simulated-based environment for teaching and learning had greatly improved the health workers HBB knowledge and skill in neonatal resuscitation; however, this knowledge was not retained at three months.

Among the points of interest is that the practical psychomotor skill and competency of the health workers improved greatly and were retained after three months. Surprisingly, the health workers retained the skill and competency at three months despite the similar major studies about retention of practical skills and competency conducted in Rwanda and Kenya that indicated that it was mostly difficult to retain the skill and competency at three to six months after the Helping Babies Training Intervention.

The persistence of the practical skill and competency among the trainees could be attributed to the implementation of quality improvement cycle during our study with a focus on the practical application of the HBB steps and problem solving. Many studies on HBB reported a decline in the knowledge and practical skill within three to six months of receiving the neonatal resuscitation training. ${ }^{12}$ Similarly, it was reported that the practical skill and competency fades faster than the knowledge. ${ }^{13}$

Our study finding confirmed that the HBB is a practical course that requires actions with periodic reinforcement of the skill through review and problem solving and self-assessment of retention and skill learned. Furthermore, an evaluation study conducted in Ghana on the retention of knowledge and practical skills and the competency of health workers at 9-12 months after training on modified neonatal resuscitation program indicated that the knowledge and skill remained stable within the period of 9-12 months post training. This mostly concurs with the retention of practical skill and competency found among the health workers from the Juba Teaching Hospital.

Table 3. Early newborn mortality.

\begin{tabular}{|c|c|c|c|c|c|c|}
\hline \multirow[t]{2}{*}{ Variable } & \multirow[b]{2}{*}{ Intervention/Control } & \multicolumn{2}{|c|}{ Before Intervention } & \multicolumn{2}{|c|}{ After Intervention } & \multirow[b]{2}{*}{ P Value } \\
\hline & & Frequency & Percent (\%) & Frequency & Percent (\%) & \\
\hline \multirow[t]{3}{*}{ Total live births } & Intervention & 1116 & 52.40 & 1112 & 53.9 & - \\
\hline & Control & 1011 & 47.50 & 950 & 46 & - \\
\hline & Total & 2127 & 99.9 & 2,062 & 99.9 & \\
\hline \multirow[t]{3}{*}{ Newborn Birth Asphyxia } & Intervention & 88 & 55.7 & 125 & 57.1 & 0.18 \\
\hline & Control & 70 & 44.3 & 94 & 42.9 & - \\
\hline & Total & 158 & & 219 & & \\
\hline \multirow[t]{3}{*}{ Newborn resuscitated using HBB } & Intervention & 0 & 00 & 124 & 98.4 & $0.001^{*}$ \\
\hline & Control & 0 & 00 & 2 & 1.6 & 0.114 \\
\hline & Total & 0 & & 126 & & \\
\hline \multirow[t]{3}{*}{ Asphyxia deaths } & Intervention & 26 & 50.9 & 4 & 30.7 & $0.001^{*}$ \\
\hline & Control & 25 & 49 & 9 & 69.2 & 0.110 \\
\hline & Total & 51 & & 13 & & \\
\hline \multirow[t]{3}{*}{ Death within 24 hours } & Intervention & 14 & 51.9 & 4 & 23.5 & $0.001^{*}$ \\
\hline & Control & 13 & 48.1 & 13 & 48.1 & 0.110 \\
\hline & Total & 27 & & 17 & & \\
\hline \multirow[t]{3}{*}{ Death after 24 hours } & Intervention & 12 & 50.0 & 9 & 33.3 & $0.000 *$ \\
\hline & Control & 12 & 50.0 & 18 & 66.7 & 0.112 \\
\hline & Total & 24 & & 37 & & \\
\hline
\end{tabular}

*Significant level at 0.05 . Rounded at 1 decimal place Tested by Pearson Chi square test $2 \times 2$ sided significance for birth asphyxia, newborn death within and after 24 hours before and after implementation. 
Many of the researchers who conducted a similar study advocated for the means of retention of knowledge, skill, and competencies among the trainees and suggested refresher training courses between the post training and implementation period..$^{14}$

During the study evaluation of the HBB training, we found out that $5.2 \%$ failed the written test at post training, and this further increased to $19 \%$ at the end of three months. Similarly, $11.9 \%$ failed the practical skills at post-test and $18.3 \%$ at three months and the passing scores were not met (Table 2). Our failure rate in the study among the health workers was similar to other studies conducted by Singhal at al indicating that health workers skills and competency for neonatal intubation and ventilation remained very limited after training our results of the failure rate among the health workers was demonstrated by other studies reporting the limited skills and competency in neonatal intubation and ventilation. ${ }^{15}$

The training intervention revealed a potential benefit of not only improving the knowledge, skills, and competency of the health workers but has also impacted on the newborn outcomes. A remarkable decline in the newborn mortality ratio due to asphyxia was noted among the intervention Hospital. The early newborn infant deaths within 24 hours due to asphyxia reduced from $51.9 \%$ to $233.5 \%$. The association between knowledge and neonatal reduction at the same period was not explored in this study. On the Global context, a few studies have demonstrated the long term of effects of the HBB training of health workers in the early neonatal outcomes. Similarly, a large before-and-after design study conducted in Tanzania showed that training in and the targeted implementation of the HBB program was associated with a significant reduction in the primary outcome of the early neonatal mortality (within 24 hours) and the rate of fresh stillbirths and early perinatal mortality. ${ }^{16}$

There was no significant increase in the ratio of early newborn mortality in the control site $(48.1 \%$ to $48.1 \%$ both pre and post implementation). Although, there was notably decreased in the ratio of early newborn deaths at intervention site, the three months period for baseline and implementation was not enough make the interpretation of the result conclusive and generalized

The strengths of our study was the use of the research instruments that was previously validated, standardized, and adapted from the American Academy of Pediatrics and was used to evaluate the HBB knowledge, skills, and competency of the health workers in the similar setting in low-income countries like Kenya, Rwanda, and
Uganda. ${ }^{17}$ Despite the study being extensive, there were many limitations to it: some due to the inherent issues with the study design used and some due to the conflict, time, and finances that had direct and indirect effects on the study result. First, the study's ability to evaluate resuscitation practices was limited by the small number of infants who required active resuscitation; however, the authors were able to demonstrate improvements in preparation for the resuscitation.

Second, the pre- and post-test design of the study with the introduction of the quality improvement cycle limited the researchers' ability to determine the effects of the HBB training alone. It also hindered them from experiencing the changes in the knowledge, skills, and competency during the implementation let alone the significant reduction of the early neonatal mortality. However, no ongoing similar intervention was performed at the hospital during our implementation.

Third: Our study did not assess the control group for the psychomotor sill and competency attributed to the prevailing security and this affected our comparison of intervention and control group at baseline.

The study strongly recommends that since the HBB training had an positive impact on the knowledge, skill, and competency of health workers in the hospital setting and in the reduction of early neonatal mortality, training must be provided by the Ministry of Health and other supporting organizations in the country particularly in rural setting where this protocol has put emphasis. The training must be conducted and an in-phased approach must be used when training the first pool of facilitators and health workers where neonatal mortality indicators are highest. It is recommended that similar research on HBB should consider assessing both groups (Intervention and Control) at baseline for knowledge, psychomotor skills and competency.

\section{Conclusions}

The study has demonstrated that the health workers from Juba Teaching Hospital in South Sudan significantly improved their knowledge, practical and competency on neonatal resuscitation after participating in a two-day training course. With the ongoing conflict, it was expected that training of health workers in HBB might not have the hypothesized impact, but this was proven to be wrong; however, the knowledge was found to decline during the three-month follow-up. Interestingly, the practical skill and competency of the health workers remained intact over the three-month period and even continued to increase strongly among the health workers evaluated. Additionally, the training and implementation had a positive effect on the survival rate of neonates evaluated in the teaching hospitals. Overall, a significant reduction in early newborn mortality rates due to asphyxia-related illness was noted after the implementation. This may prove directly replicable in other similar settings, not only in South Sudan, but also in other low-income countries.

\section{References}

1. Countdown Coverage Writing Group. Countdown to 2015 for maternal, newborn, and child survival. Lancet 2008;371:1274-8.

2. Goldenberg RL, McClure EM, Bann CM. The relationship of intrapartum and antepartum stillbirth rates to measures of obstetric care in developed and developing countries. Acta Obstet Gynecol Scand 2007;86;1303-9.

3. World Health Organization. Making pregnancy safer: the critical role of the skilled attendant. A joint statement by WHO, ICM and FIGO. Geneva: World Health Organization; 2005.

4. Lawn J, Kerber K, Enweronu-Laryea C, Cousens S. 3.6 million Neonatal deaths--what is progressing and what is not? Sem Perinatol 2010;34:371-86.

5. Save the Children. Ending Newborn Deaths. London, UK; 2014.

6. UNICEF. Committing to Child Survival: A Promise Renewed Progress Report 2013. New York: UNICEF; 2013.

7. World Health Organization. World health statistics. WHO; 2010.

8. Lawn JE, Lee AC, Kinney M, et al. Two million intrapartum-related stillbirths and neonatal deaths: where, why, and what can be done? Int J Gynecol Obstet 2009;107:S5-18,19.

9. Opiyo N, English M. In-service training for health professionals to improve seriously ill newborn or child in low and middle-income countries (review). Cochrane Database Syst Rev 2010;4.

10. Singhal N, Lockyer J, Fidler H, et al. Helping Babies Breathe: Global neonatal resuscitation program development and formative educational evaluation. Resuscitation 2012;83:90-6.

11. American Academy of Pediatrics Helping Babies Breathe: 2014. Available from http://www.helpingbabiesbreathe.org/implementationguide.ht $\mathrm{ml}$

12. Trevisanuto D, Ferrarese P, Cavicchioli $\mathrm{P}$, et al. Knowledge gained by pediatric 
residents after neonatal resuscitation program courses. Paediatr Anaesth 2005; 15:944-7.

13. Carlo WA, Wright LL, Chomba E, et al. Educational impact of the neonatal resuscitation program in low-risk delivery centers in a developing country. J Pediatr 2009;154:504-8e5.

14. Kaczorowski J, Levitt C, Hammond M, et al. Retention of neonatal resuscitation skills and knowledge: a randomized controlled trial. Fam Med 1998;30:70511 .

15. Singhal N, Lockyer J, Fidler H, et al. Helping Babies Breathe: Global neonatal resuscitation program development and formative educational evaluation. Resuscitation 2012;83:90-6.

16. Georgina M, Augustine M, Donan M, et al. Newborn mortality and fresh still- birth rates in Tanzania after Helping Babies Training. Pediatrics 2013;131:2.

17. Korioth T. Helping Babies Breathe: New global program to boost newborn survival rates. AAP News 2010;31. Available from: http:// aapnews.aappublications.org/content/31/8/1.1.full.p df + html. Accessed: June 24, 2017. 